\title{
Trapezoidal Approximation of a Fuzzy Number Preserving the Expected Interval and Including the Core
}

\author{
B. Asady \\ Department of Mathematics, Islamic Azad University, Arak Branch, Arak, Iran \\ Email: babakmz2002@yahoo.com,b-asadi@iau-arak.ac.ir
}

Received October 11, 2012; revised November 15, 2012; accepted November 28, 2012

\begin{abstract}
In this paper, we introduce a method to obtain the nearest trapezoidal approximation of fuzzy numbers so that preserving conditions expect interval and include the core of a fuzzy number.
\end{abstract}

Keywords: Trapezoidal Approximation; Fuzzy Number; Fuzzy Partition; Fuzzy Approximation; Trapezoidal Fuzzy Numbers

\section{Introduction}

Trapezoidal fuzzy intervals are often used in practice. An interesting problem is to approximate general fuzzy intervals by means of trapezoidal ones, so as to simplify calculations. The investigations in this area were started by Ma et al. [1] that proposed the symmetric triangular approximation. Actually, symmetric triangular approximation is a particular case of the trapezoidal approximation that was discussed by many authors including Abbasbandy, Amirfakhrian [2], Abbasbandy and Asady [3], Coroianu [4], Ban [5-8], Grzegorzewski [9-11], Grzegorzewski and Mrowka [12,13], Grzegorzewski, K. Pasternak-Winiarska [14,15] and Yeh [16-21] and other methods same as [22-27]. Although, there are many scholars who have investigated interval, triangular and trapezoidal approximation of fuzzy numbers, but the result of approximation is not always a fuzzy number, sometimes it is not a fuzzy set. For example Grzegorzewski and Mrowka proposed in [12] a method to find the nearest (with respect to a well-known metric between fuzzy numbers) trapezoidal approximation operator that preserving the expected interval. Unfortunately, there was a gap in the suggested solution which was later improved by Grzegorzewski and Mrowka [13] and finally solved by Ban [7] and Yeh [18]. In this paper, we combine the ideas proposed in papers [3,12], so that preserving conditions expect interval and include the core of a fuzzy number. In Section 2, we recall some fundamental results on fuzzy numbers. Trapezoidal approximation and examples are in Section 3.

\section{Preliminaries}

Definition 1. (cf. [28]) A fuzzy number is a fuzzy set like
$A: R \rightarrow I=[0,1]$ which satisfies:

1) $A$ is the strictly quasi-convex,

2) $A(x)=0$ outside some interval $[c, d]$,

3) There are real numbers $a, b$ such that $c \leq a \leq b \leq d$ and

a) $A(x)$ is monotonic increasing on $[c, a]$,

b) $A(x)$ is monotonic decreasing on $[b, d]$,

c) $A(x)=1, a \leq x \leq b$.

The set of all these fuzzy numbers is denoted by $F(R)$. The $\alpha$-cut, $\alpha \in 2] 0,1]$, of a fuzzy number $A$ is a crisp set defined as

$$
A \alpha=\{x \in R: A(x) \geq \alpha\} .
$$

Every $\alpha$-cut of a fuzzy number $A$ is a closed interval $A \alpha=[A L(\alpha), A U(\alpha)]$, where

$$
\begin{aligned}
& A L(\alpha)=\inf \{x \in R: A(x) \geq \alpha\}, \\
& A U(\alpha)=\sup \{x \in R: A(x) \geq \alpha\} .
\end{aligned}
$$

The set of all elements that have a nonzero degree of membership in $A$ is called the support of $A$ [27], i.e.

$$
\operatorname{supp} A=\{x \in R: A(x)>0\} .
$$

Definition 2. (complement) The complement of a fuzzy number $A$ is defined as

$$
(\neg A)(x)=1-A(x) .
$$

The pair of functions $(A L, A U)$ gives a parametric representation of fuzzy number $A$ (see [29]). Another important type of fuzzy numbers was introduced in [23] as follows.

Let $a_{1}, a_{2}, a_{3}, a_{4} \in R$ such that $a_{1} \leq a_{2} \leq a_{3} \leq a_{4}$. A fuzzy number defined as 


$$
A(x)= \begin{cases}0 & \text { if } x \leq a_{1} \\ \left(\frac{x-a_{1}}{a_{2}-a_{1}}\right)^{l} & \text { if } a_{1} \leq x \leq a_{2} \\ 1 & \text { if } a_{2} \leq x \leq a_{3} \\ \left(\frac{a_{4}-x}{a_{4}-a_{3}}\right)^{r} & \text { if } a_{3} \leq x \leq a_{4} \\ 0 & \text { if } x \geq a_{4}\end{cases}
$$

where $l, r>0$, is denoted by $A=\left(a_{1}, a_{2}, a_{3}, a_{4}\right) \mathrm{lr}$ and if $r=1$ by $A=\left(a_{1}, a_{2}, a_{3}, a_{4}\right) r$. Its parametric form is

$$
\begin{aligned}
A_{\alpha} & =\left[A_{L}(\alpha), A_{U}(\alpha)\right] \\
& =\left[a_{1}+\alpha^{\frac{1}{l}}\left(a_{2}-a_{1}\right), a_{4}-\alpha^{\frac{1}{r}}\left(a_{4}-a_{3}\right)\right], \alpha \in[0,1]
\end{aligned}
$$

A popular fuzzy number is the trapezoidal fuzzy number, completely characterized by Equation (1) when $l=r=1$, denoted by $A=\left(a_{1}, a_{2}, a_{3}, a_{4}\right)$. We denoted by $F(R)$ the set of all fuzzy numbers and $F T(R)$ the set of all trapezoidal fuzzy numbers.

Definition 3. For arbitrary fuzzy numbers $A$, $A \alpha=[A L(\alpha), A U(\alpha)]$ and $B$, $B \alpha=[B L(\alpha), B U(\alpha)]$ the quantity

$$
\begin{aligned}
D(A, B)= & \sqrt{\int_{0}^{1}\left(A_{L}(\alpha)-B_{L}(\alpha)\right)^{2} \mathrm{~d} \alpha} \\
& +\int_{0}^{1}\left(A_{U}(\alpha)-B_{U}(\alpha)\right)^{2} \mathrm{~d} \alpha
\end{aligned}
$$

is the distance between $A$ and $B,[3,24,25]$. The function $D(A, B)$ is a metric in $F(R)$ and $(F(R), D)$ is a complete metric space. The expected interval $E I(A)$ of a fuzzy number $A A \alpha=[A L(\alpha), A U(\alpha)]$ introduced independently by Dubois and Prade [30] and Heilpern [31]. It is defined by

$$
\begin{aligned}
E I(A) & =\left[E^{*}(A), E^{*}(A)\right] \\
& =\left[\int_{0}^{1} A_{L}(\alpha) \mathrm{d} \alpha, \int_{0}^{1} A_{u}(\alpha) \mathrm{d} \alpha\right]
\end{aligned}
$$

Grzegorzewski [9] shows that the interval $E I(A)$ is the nearest interval to the fuzzy number $A$. Hence in [31] the expected value of a fuzzy number $A$ defined following as

$$
E V(A)=\frac{1}{2}\left(E_{*}(A)+E^{*}(A)\right)
$$

and B. Asady and M. Zendehnam in [32] show that $E V(A)$ is the best approximation of a fuzzy number $A$. Finally, let us recall that $\operatorname{core} A=\{x R: A(x)=1\}$.

\section{Trapezoidal Approximation of Fuzzy Numbers}

Suppose we want to approximate a fuzzy number by a trapezoidal fuzzy number. Thus, we use an operator $T: F(R) \rightarrow F T(R)$ which transforms fuzzy numbers into family of trapezoidal fuzzy number.

Abbasbandy and Asady [3] considered a trapezoidal approximation that includes the $\alpha$-cut superset, i.e.,

$$
\left(T_{1}\right) \operatorname{core}(A) \subseteq \operatorname{core} T(A)
$$

Grzegorzewski and Mrówka [12] said that an operator $T: F(R) \rightarrow F T(R)$ fulfills the criterion if for any fuzzy number $A$ its output value $T(A)$ preserves the expected interval, i.e.,

$$
\left(T_{2}\right) E I(A)=E I(T(A))
$$

In this part, we combine ideas proposed in the papers $[3,12]$ to obtain the nearest trapezoidal fuzzy number respect to the original fuzzy number so that it preserves $T_{1}$ and $T_{2}$ conditions. Since, any $x$ belongs to the core $A$ of a fuzzy number $\mathrm{A}$ if and only if it does not belong to the complement of $\mathrm{A}$

$$
(x \in \operatorname{core} A \Leftrightarrow x \notin \neg A)
$$

Therefore, for preserving of these points we consider core $A \subseteq \operatorname{core} T(A)$. Also, we are going to preserve the expected interval of the fuzzy number that this additional requirement by the significant role of the expected interval is in many situations and applications (see, e.g., [5-11, 33]. Additionally, the propose approach can provide decision makers with a new alternative to trapezoidal approximation of fuzzy numbers.

Now, given a fuzzy number $B$ with $\alpha$-cut set $[B L(\alpha), B U(\alpha)]$, the problem is to find a trapezoidal fuzzy number $T(B)=\left(t_{1}, t_{2}, t_{3}, t_{4}\right)$ which is the nearest to $B$ with respect to metric $D$ and preserves the conditions $T_{1}$ and $T_{2}$ i.e. we have

$$
\begin{aligned}
\min \{D(B, T(B))\}= & \left\{\left[\int_{0}^{1}\left(B_{L}(\alpha)-T(B)_{L}(\alpha)\right)^{2} \mathrm{~d} \alpha\right.\right. \\
& \left.\left.+\int_{0}^{1}\left(B_{U}(\alpha)-T(B)_{U}(\alpha)\right)^{2} \mathrm{~d} \alpha\right]^{\frac{1}{2}}\right\}
\end{aligned}
$$

Subject to

$$
\begin{gathered}
{\left[t_{2}, t_{3}\right] \subseteq\left[B_{L}(1), B_{U}(1)\right],} \\
{\left[\frac{t_{1}+t_{2}}{2}, \frac{t_{3}+t_{4}}{2}\right]=\left[\int_{0}^{1} B_{L}(\alpha) \mathrm{d} \alpha, \int_{0}^{1} B_{U}(\alpha) \mathrm{d} \alpha\right],}
\end{gathered}
$$

As, in order to minimize $D(B, T(B))$ it suffices to minimize function 


$$
\begin{aligned}
F\left(h_{1}, h_{2}\right)= & D^{2}(B, T(B)) \\
= & \int_{0}^{1}\left(B_{L}(\alpha)-\left(t_{1}+\left(t_{2}-t_{1}\right) \alpha\right)\right)^{2} \mathrm{~d} \alpha \\
& +\int_{0}^{1}\left(B_{U}(\alpha)-\left(t_{4}+\left(t_{4}-t_{3}\right) \alpha\right)\right)^{2} \mathrm{~d} \alpha,
\end{aligned}
$$

with respect to $h_{1}$ and $h_{2}$. Subject to

$$
t_{2}=B_{L}(1)-h_{1}^{2}
$$

and

$$
t_{3}=B_{U}(1)-h_{2}^{2}
$$

and

$$
t_{1}=2 \int_{0}^{1} B_{L}(\alpha) \mathrm{d} \alpha-t_{2},
$$

and

$$
t_{4}=2 \int_{0}^{1} B_{U}(\alpha) \mathrm{d} \alpha-t_{3},
$$

Clearly, with note to Equations (8), (9), we can say the conditions (11)-(14) are suitable for finding the nearest trapezoidal fuzzy number $T(B)$ to a fuzzy number $B$ with the conditions as the expected interval and core $B \alpha$ core $(T(B))$ are preserved. Theorem 1: Let $B$ with $\alpha$-cut set $[B L(\alpha), B U(\alpha)]$, be a fuzzy number and

$$
\begin{aligned}
d_{1}= & B_{L}(1)-\int_{0}^{1} B_{L}(\alpha) \mathrm{d} \alpha \\
& -3 \int_{0}^{1} B_{L}(\alpha)(2 \alpha-1) \mathrm{d} \alpha, \\
d_{2}= & -B_{U}(1)+\int_{0}^{1} B_{U}(\alpha) \mathrm{d} \alpha \\
& +3 \int_{0}^{1} B_{U}(\alpha)(2 \alpha-1) \mathrm{d} \alpha,
\end{aligned}
$$

Case 1: If $d_{1} \leq 0$ and $d_{2} \leq 0$ then, optimal solution of problem (10) is

and consequently, we have

$$
h_{1}=h_{2}=0
$$

$$
\begin{aligned}
& t_{1}=-B_{L}(1)+2 \int_{0}^{1} B_{L}(\alpha) \mathrm{d} \alpha, \\
& t_{2}=B_{L}(1), \\
& t_{3}=B_{U}(1), \\
& t_{4}=-B_{U}(1)+2 \int_{0}^{1} B_{U}(\alpha) \mathrm{d} \alpha
\end{aligned}
$$

Case 2: If $d_{1}>0$ and $d_{2} \leq 0$ then

$$
\begin{aligned}
& h_{1}=\sqrt{h_{1}}, h_{2}=0, \\
& t_{1}=4 \int_{0}^{1} B_{L}(\alpha) \mathrm{d} \alpha-6 \int_{0}^{1} \alpha B_{L}(\alpha) \mathrm{d} \alpha, \\
& t_{2}=-2 \int_{0}^{1} B_{L}(\alpha) \mathrm{d} \alpha+6 \int_{0}^{1} \alpha B_{L}(\alpha) \mathrm{d} \alpha, \\
& t_{3}=B_{U}(1), \\
& t_{4}=-B_{U}(1)+2 \int_{0}^{1} B_{U}(\alpha) \mathrm{d} \alpha,
\end{aligned}
$$

Case 3: If $d_{1} \leq 0$ and $d_{2}>0$ then

$$
\begin{aligned}
& h_{1}=0, h_{2}=\sqrt{d_{2}}, \\
& t_{1}=-B_{L}(1)+2 \int_{0}^{1} B_{L}(\alpha) \mathrm{d} \alpha, \\
& t_{2}=B_{L}(1), \\
& t_{3}=-2 \int_{0}^{1} B_{U}(\alpha) \mathrm{d} \alpha+6 \int_{0}^{1} \alpha B_{U}(\alpha) \mathrm{d} \alpha, \\
& t_{4}=4 \int_{0}^{1} B_{U}(\alpha) \mathrm{d} \alpha-6 \int_{0}^{1} \alpha B_{U}(\alpha) \mathrm{d} \alpha,
\end{aligned}
$$

Case 4: If $d_{1}>0$ and $d_{2}>0$ then

$$
\begin{aligned}
& h_{1}=\sqrt{d_{1}}, h_{2}=\sqrt{d_{2}} \\
& t_{1}=4 \int_{0}^{1} B_{L}(\alpha) \mathrm{d} \alpha-6 \int_{0}^{1} \alpha B_{L}(\alpha) \mathrm{d} \alpha, \\
& t_{2}=-2 \int_{0}^{1} B_{L}(\alpha) \mathrm{d} \alpha+6 \int_{0}^{1} \alpha B_{L}(\alpha) \mathrm{d} \alpha, \\
& t_{3}=-2 \int_{0}^{1} B_{U}(\alpha) \mathrm{d} \alpha+6 \int_{0}^{1} \alpha B_{U}(\alpha) \mathrm{d} \alpha, \\
& t_{4}=4 \int_{0}^{1} B_{U}(\alpha) \mathrm{d} \alpha-6 \int_{0}^{1} \alpha B_{U}(\alpha) \mathrm{d} \alpha,
\end{aligned}
$$

Proof: In order to minimize $F\left(h_{1}, h_{2}\right)$ it suffices to minimize function $F 2\left(h_{1}, h_{2}\right)=f\left(h_{1}, h_{2}\right)$ with the partial derivatives

$$
\left\{\begin{array}{l}
\frac{\partial f\left(h_{1}, h_{2}\right)}{\partial h_{1}} \\
=\frac{4}{3} h_{1}\left(h_{1}^{2}-B_{L}(1)+\int_{0}^{1} B_{L}(\alpha) \mathrm{d} \alpha+3 \int_{0}^{1} B_{L}(\alpha)(2 \alpha-1) \mathrm{d} \alpha\right) \\
\frac{\partial f\left(h_{1}, h_{2}\right)}{\partial h_{2}} \\
=\frac{4}{3} h_{2}\left(h_{2}^{2}+B_{U}(1)-\int_{0}^{1} B_{U}(\alpha) \mathrm{d} \alpha-3 \int_{0}^{1} B_{U}(\alpha)(2 \alpha-1) \mathrm{d} \alpha\right)
\end{array}\right.
$$




$$
\left\{\begin{array}{l}
\frac{\partial^{2} f\left(h_{1}, h_{2}\right)}{\partial h_{1}^{2}}=4 h_{1}^{2}-\frac{4}{3} d_{1} \\
\frac{\partial^{2} f\left(h_{1}, h_{2}\right)}{\partial h_{2}^{2}}=4 h_{2}^{2}-\frac{4}{3} d_{2} \\
\frac{\partial^{2} f\left(h_{1}, h_{2}\right)}{\partial h_{1} \partial h_{2}}=0,
\end{array}\right.
$$

system (10) is $h_{1}=h_{2}=0$ and the point $(0,0)$ minimizes the function $F$ in the Equation (10). Because $F\left(h_{1}, h_{2}\right)$ is lower bounded function and has only a critical point. Also, with note to Equations (11)-(14) and optimal solution $h_{1}=h_{2}=0$ formula (17) is correct.

2) If $d_{1}>0$ and $d_{2} \leq 0$ are satisfied then the solutions of the system (10) are

Case 1: $h_{1}=0, h_{2}=0$,

Case 2: $h_{1}=\sqrt{d_{1}}, \quad h_{2}=0$

Moreover, since

$$
\left\{\begin{array}{l}
\frac{\partial^{2} f(0,0)}{\partial h_{1}^{2}}<0, \\
\frac{\partial^{2} f(0,0)}{\partial h_{2}^{2}} \geq 0, \\
\frac{\partial^{2} f\left(\sqrt{d_{1}}, 0\right)}{\partial h_{1}^{2}}=\frac{8}{3} d_{1}>0, \\
\frac{\partial^{2} f\left(\sqrt{d_{1}}, 0\right)}{\partial h_{2}^{2}}=-\frac{4}{3} d_{2} \geq 0,
\end{array}\right.
$$

therefore case 2 such that $h_{1}=\sqrt{d_{1}}, h_{2}=0$ minimizes the function $F$. Because the Hessian matrix for it is positive definite and for case 1 is not positive definite. Also, with note to Equations (11)-(14) and $h_{1}=\sqrt{d_{1}}, h_{2}=0$ Formulae (18) are satisfied.

3) Proof is similar with 2)

4) If $d_{1}>0$ and $d_{2}>0$ are satisfied then the solutions of the system (10) are given by

Case 1: $h_{1}=0, h_{2}=0$;
Case 2: $h_{1}=0, h_{2}=\sqrt{d_{2}}$;

Case 3: $h_{1}=\sqrt{d_{1}}, h_{2}=0$;

Case 4: $h_{1}=\sqrt{d_{1}}, h_{2}=\sqrt{d_{2}}$.

Because the Hessian matrix for of case 4 is positive definite and for others cases are not positive definite, then the solution of case 4 is minimizer and formulae (20) is correct. 2 Now we compare our method with the other works $[3,7,20]$ in following examples.

Example 1. [7] Let us consider the fuzzy number $A=(1,2,3,30) \times 2$, in the parametric forms,

$$
A_{L}(\alpha)=1+\sqrt{\alpha}, A_{U}(\alpha)=30-27 \sqrt{\alpha}, \alpha \in[0,1] .
$$

By substitute above equations on the (2) and (3) equations, we obtain the expect interval and core of the fuzzy number A same as follows

$$
E I(A)=\left[\frac{5}{3}, 12\right] \text {, core }(A)=[2,3]
$$

Also, trapezoidal approximation of it by the other methods and proposed method is shown in Table 1.

Clearly, in Table 1, by Ban and Yeh methods, we have

$$
E I(A)=E I(T(A))
$$

and

$$
\text { core } A \varsubsetneqq \operatorname{core} T(A) \text {. }
$$

and, by Abbasbandy and Asady method, get

$$
E I(A) \neq E I(T(A))
$$

and

$$
\operatorname{core} A \subseteq \operatorname{core} T(A)
$$

Consequently, with note above results proposed method preserved $\mathrm{T} 1$ and $\mathrm{T} 2$ conditions (see Figures 1-4).

Example 2. Consider the fuzzy number $B=(-10,10,10,14)_{41 / 3}$ that the parametric form of it is as follows

Table 1. Comparative results of example 1.

\begin{tabular}{cccc}
\hline Authors & The trapezoidal approximation of fuzzy numbers $A$ & $E I(T(A))$ & $\operatorname{core} T(A)$ \\
\hline Ban & $(5 / 3,5 / 3,5 / 3,67 / 3)$ & {$\left[\frac{5}{3}, 12\right]$} & $\frac{5}{3}$ \\
Yeh & $(47 / 35,71 / 35,78 / 35,726 / 35)$ & {$\left[\frac{5}{3}, 12\right]$} & {$\left[\frac{71}{35}, \frac{78}{35}\right]$} \\
Abbasbandy Asady & $(13 / 10,2,3,219 / 10)$ & {$[2,3]$} \\
Proposed method & $(3 / 4,2,3,21)$ & {$\left[\frac{5}{20}, 12\right]$} & {$[2,3]$} \\
\hline
\end{tabular}




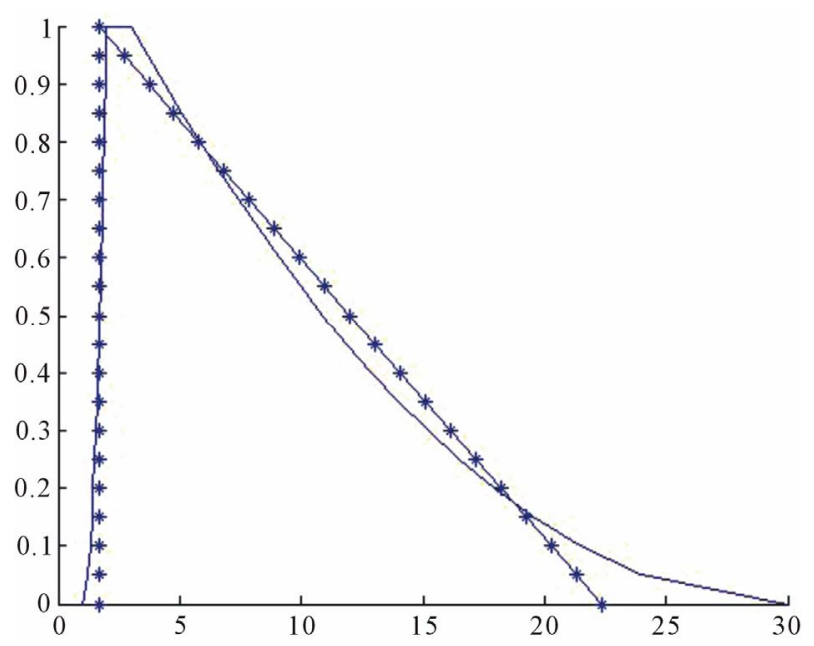

Figure 1. The trapezoidal approximation of $A$ by Ban method.

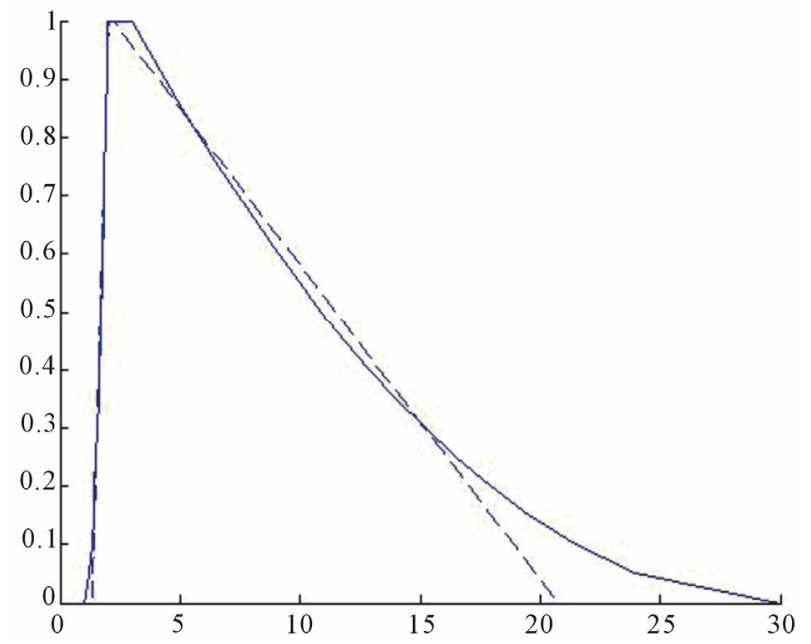

Figure 2. The trapezoidal approximation of $A$ by Yeh method.

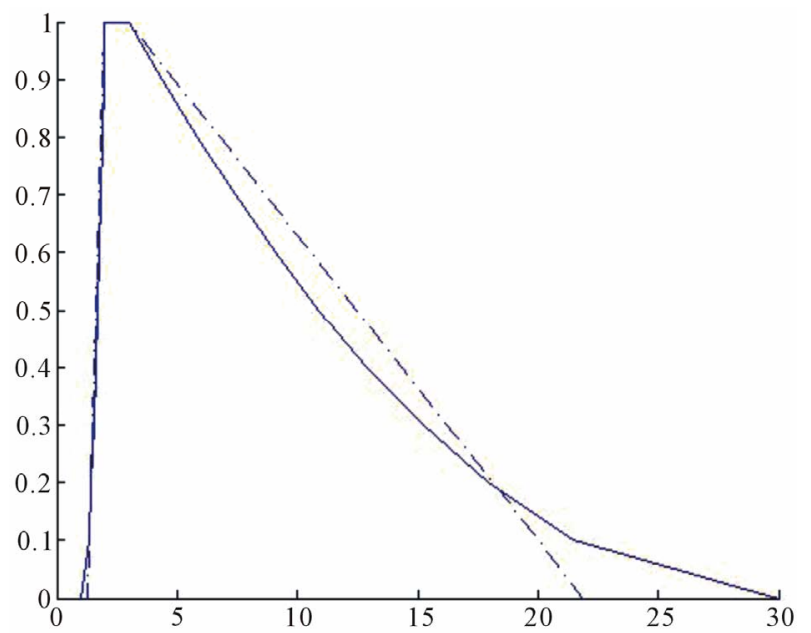

Figure 3. The trapezoidal approximation of $A$ by Abbasbandy and Asady method.

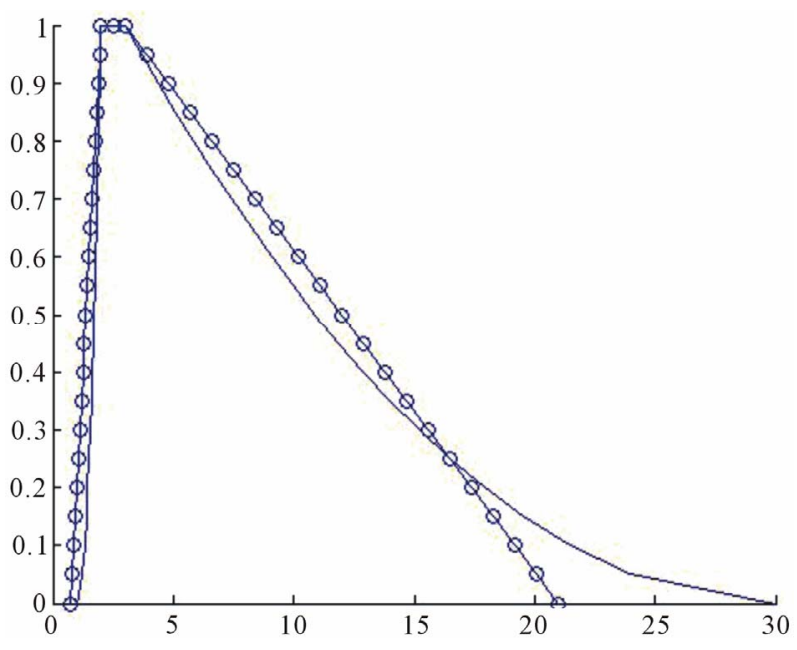

Figure 4. The trapezoidal approximation of $A$ by prposed method.

$$
B L(\alpha)=-10+20 \alpha^{1 / 4}, B U(\alpha)=14-4 \alpha^{3} .
$$

Clearly, by Equations (2) and (3) the expect interval and core of it are same as follows

$$
E I(B)=[6,13] \text { and core } B=\left[10, \frac{56}{5}\right] \text {. }
$$

Also, trapezoidal approximation of the fuzzy number $B$ by the other methods and proposed method is shown in Table 2.

Clearly, in Table 2 by Ban and Yeh methods, core $B \mp \operatorname{core} T(B)$ and even $\operatorname{core} B \cap \operatorname{core} T(B)=\varnothing$ (see Figures 5 and 6). But, for Abbasbandy and Asady method, we get $E I(B) \neq E I(T(B))$. Finally, the results of proposed method are

$$
E I(A)=E I(T(A))
$$

and $\operatorname{core} A \subseteq \operatorname{core} T(A)$. Therefore, we can say that proposed method preserves $T 1$ and $T 2$ conditions in example 2. (please see Figures 7 and 8 ).

Theorem 3. Trapezoidal approximation satisfies the following properties.

1) Translation invariance: $\forall z \in R, \forall A \in F(R)$ : then we have $T(A+z)=T(A)+z$.

2) Scale invariance: $\forall z \in R, \forall A \in F(R)$ : then we have $T(z A)=z T(A)$.

Proof. Proof is similar to proof of Theorem 12 in [7].

Theorem 4. Trapezoidal approximation satisfies the identity property.

Proof. Let $B=\left(b_{1}, b_{2}, b_{3}, b_{4}\right)$ be a trapezoidal fuzzy number. By Equations (2) and (3), we get $d_{1}=\frac{t_{2}-t_{1}}{2}$, $d_{2}=\frac{t_{4}-t_{3}}{2}$.

Therefore, $d_{1}$ and $d_{2}$ are positive and conditions in the 
Table 2. Comparative results of Example 2.

\begin{tabular}{|c|c|c|c|}
\hline Authors & The trapezoidal approximation of fuzzy numbers $B$ & $E I(T(B))$ & $\operatorname{core} T(B)$ \\
\hline Ban & $T B(B)=(11 / 15,169 / 15,169 / 15,221 / 15)$ & {$[6,13]$} & $\frac{169}{15}$ \\
\hline Yeh & $T_{Y}(B)=(90 / 39,410 / 39,54 / 5,78 / 5)$ & {$[6,13]$} & {$\left[\frac{410}{39}, \frac{54}{5}\right]$} \\
\hline Abbasbandy, Asady & $T_{A}(B)=(4 / 3,10,56 / 5,74 / 5)$ & {$\left[\frac{17}{3}, 13\right]$} & {$\left[10, \frac{56}{5}\right]$} \\
\hline Proposed method & $T_{p}(B)=(2,10,56 / 5,74 / 5)$ & {$[6,13]$} & {$\left[10, \frac{56}{5}\right]$} \\
\hline
\end{tabular}

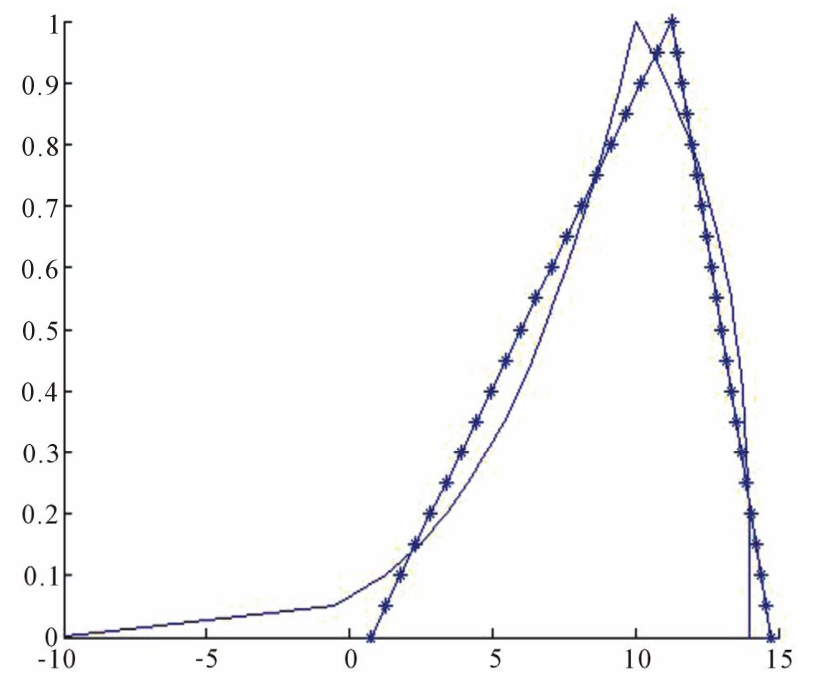

Figure 5. The trapezoidal approximation of $B$ by Ban method.

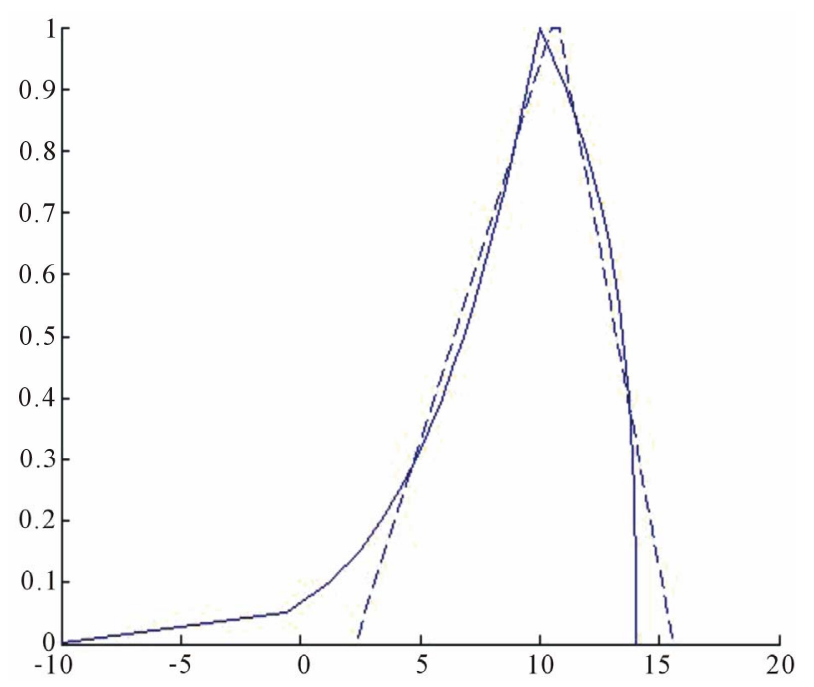

Figure 6. The trapezoidal approximation of $B$ by Yeh method.

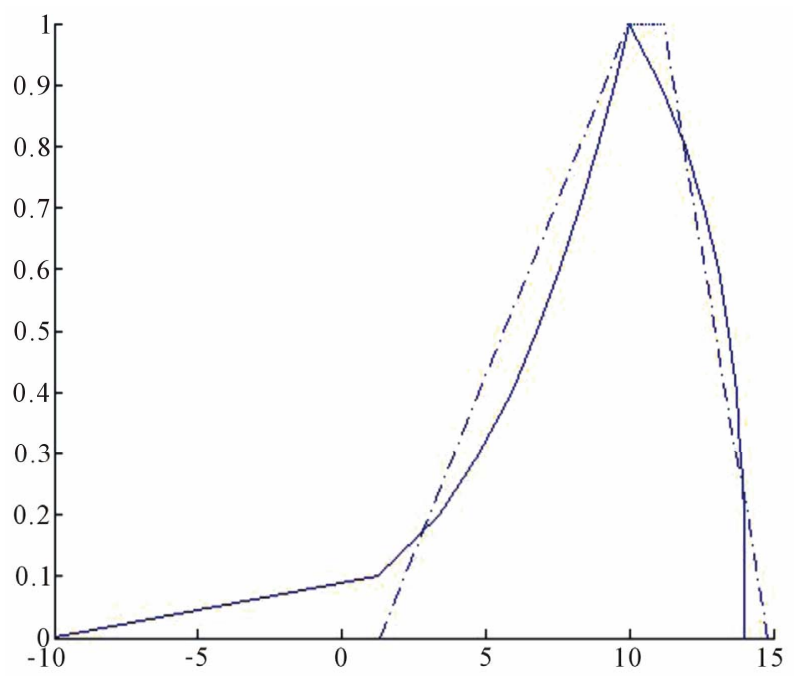

Figure 7. The trapezoidal approximation of $B$ by Abbasbandy and Asady method.

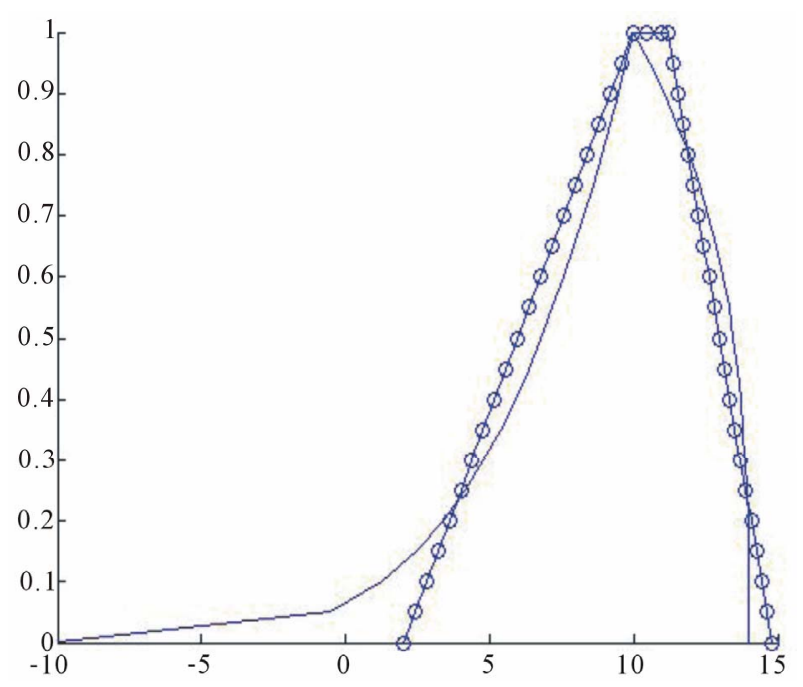

Figure 8. The trapezoidal approximation of $B$ by proposed method. 
Case 4) are satisfied, Theorem 1 is applicable. We obtain $t_{k}=b_{k}, k \in\{1,2,3,4\}$.

\section{Conclusion}

In this paper, we have been suggested an interesting approach to trapezoidal approximation of general fuzzy numbers. The proposed method leads to the trapezoidal fuzzy number which is the best one with respect to a certain measure of distance between fuzzy numbers, $D(u, v)$.

\section{REFERENCES}

[1] Ming Ma, A. Kandel and M. Friedman, "A New Approach for defuzzification,” Fuzzy Sets and Systems, Vol. 111, No. 3, 2000, pp. 351-356. doi:10.1016/S0165-0114(98)00176-6

[2] S. Abbasbandy and M. Amirfakhrian, "The Nearest Approximation of a Fuzzy Quantity in Parametric Form," Applied Mathematics and Computation, Vol. 172, No. 1, 2006, pp. 624-632. doi:10.1016/j.amc.2005.02.019

[3] S. Abbasbandy and B. Asady, "The Nearest Trapezoidal Fuzzy Number of a Fuzzy Quantity,” Applied Mathematics and Computation, Vol. 156, No. 2, 2004, pp. 381-386.

[4] L. Coroianu, "Best Lipschitz Constant of the Trapezoidal Approximation Operator Preserving the Expected Interval,” Fuzzy Sets and Systems, Vol. 165, No. 1, 2011, pp. 81-97. doi:10.1016/j.fss.2010.10.004

[5] A. I. Ban, "On the Nearest Parametric Approximation of Fuzzy Number-Revisited,” Fuzzy Sets and Systems, Vol. 166, No. 21, 2009, pp. 3027-3047. doi:10.1016/j.fss.2009.05.001

[6] A. I. Ban, "Triangular and Parametric Approximations of a Fuzzy Number-Inadvertences and Corrections,” Fuzzy Sets and Systems, Vol. 160, No. 1, 2009, pp. 3048-3058. doi:10.1016/j.fss.2009.04.003

[7] A. I. Ban, "Approximation of Fuzzy Numbers by Trapezoidal Fuzzy Numbers Preserving the Expected Interval,” Fuzzy Sets and Systems, Vol. 159, No. 11, 2008, pp. 13271344. doi:10.1016/j.fss.2007.09.008

[8] A. I. Ban, L. Coroianu and P. Grzegorzewski, "Trapezoidal Approximation and Aggregation,” Fuzzy Sets and Systems, Vol. 177, No. 1, 2011, pp. 45-59. doi:10.1016/j.fss.2011.02.016

[9] P. Grzegorzewski, "Nearest Interval Approximation of a Fuzzy Number,” Fuzzy Sets and Systems, Vol. 130, No. 3, 2002, pp. 321-330. doi:10.1016/S0165-0114(02)00098-2

[10] P. Grzegorzewski, “Trapezoidal Approximations of Fuzzy Numbers Preserving the Expected Interval-Algorithms and Properties,” Fuzzy Sets and Systems, Vol. 159, No. 11, 2008, pp. 1354-1364. doi:10.1016/j.fss.2007.12.001

[11] P. Grzegorzewski, “Algorithms for Trapezoidal Approximations of Fuzzy Numbers Preserving the Expected Interval, In: B. Bouchon-Meunier, L. Magdalena, M. OjedaAciego, J.-L. Verdegay, R. R. Yager, Eds., Foundations of Reasoning under Uncertainty, Springer, Berlin, 2010, pp. 85-98. doi:10.1007/978-3-642-10728-3_5

[12] P. Grzegorzewski and E. Mrówka, “Trapezoidal Approximations of Fuzzy Numbers,” Fuzzy Sets and Systems, Vol. 153, No. 1, 2005, pp. 115-135. doi:10.1016/j.fss.2004.02.015

[13] P. Grzegorzewski and E. Mrówka, “Trapezoidal Approximations of Fuzzy Numbers-Revisited,” Fuzzy Sets and Systems, Vol. 158, No. 7, 2007, pp. 757-768. doi:10.1016/j.fss.2006.11.015

[14] P. Grzegorzewski and K. Pasternak-Winiarska, "Weighted Trapezoidal Approximations of Fuzzy Numbers,” In: J. P. Carvalho, D. Dubois, U. Kaymak, J. M. C. Sousa (Eds.), Proceedings of the Joint 2009 International Fuzzy Systems Association World Congress and 2009 European Society of Fuzzy Logic and Technology Conference, Lisbon, 2009, pp. 1531-1534.

[15] P. Grzegorzewski and K. Pasternak-Winiarska, "Bi-Symmetrically Weighted Trapezoidal Approximations of Fuzzy Numbers," In: Proceedings of 9th International Conference on Intelligent Systems Design and Applications ISDA 2009, Pisa, 30 November-2 December 2009, pp. 318-323. doi:10.1109/ISDA.2009.138

[16] R. R. Yager and D. P. Filev, "SLIDE: A Simple Adaptive Defuzzification Method," IEEE Transactions on Fuzzy Systems, Vol. 1, No. 1, 1993, pp. 69-78. doi:10.1109/TFUZZ.1993.390286

[17] C.-T. Yeh, “A Note on Trapezoidal Approximations of Fuzzy Numbers,” Fuzzy Sets and Systems, Vol. 158, No. 7, 2007, pp. 747-754. doi:10.1016/j.fss.2006.11.017

[18] C.-T. Yeh, "On Improving Trapezoidal and Triangular Approximations of Fuzzy Numbers,” International Journal of Approximate Reasoning, Vol. 48, No. 1, 2008, pp. 297-313. doi:10.1016/j.ijar.2007.09.004

[19] C.-T. Yeh, “Trapezoidal and Triangular Approximations Preserving the Expected Interval,” Fuzzy Sets and Systems, Vol. 159, No. 11, 2008, pp. 1345-1353. doi:10.1016/j.fss.2007.09.010

[20] C.-T. Yeh, "Weighted Trapezoidal and Triangular Approximations of Fuzzy Numbers,” Fuzzy Sets and Systems, Vol. 160, No. 21, 2009, pp. 3059-3079. doi:10.1016/j.fss.2009.05.008

[21] W. Y. Zeng and H. X. Li, "Weighted Triangular Approximation of Fuzzy Numbers," International Journal of Approximate Reasoning, Vol. 46, No. 1, 2007, pp. 137150. doi:10.1016/j.ijar.2006.11.001

[22] T. Allahviranloo and M. A. Firozja, "Note on 'Trapezoidal Approximation of Fuzzy Numbers',” Fuzzy Sets and Systems, Vol. 158, No. 7, 2007, pp. 755-756. doi:10.1016/j.fss.2006.10.017

[23] S. Bodjanova, "Median Value and Median Interval of a Fuzzy Number,” Information Sciences, Vol. 172, No. 1-2, 2005, pp. 73-89. doi:10.1016/j.ins.2004.07.018

[24] M. Ma, A. Kandel and M. Friedman, "Correction to 'A New Approach for Defuzzification',” Fuzzy Sets and Systems, Vol. 128, No. 1, 2002, pp. 133-134. doi:10.1016/S0165-0114(01)00248-2

[25] D. Dubois and H. Prade, "The Mean Value of a Fuzzy 
Number,” Fuzzy Sets and Systems, Vol. 24, No. 3, 1987, pp. 279-300. doi:10.1016/0165-0114(87)90028-5

[26] E. N. Nasibov and S. Peker, "On the Nearest Parametric Approximation of a Fuzzy Number,” Fuzzy Sets and Systems, Vol. 159, No. 11, 2008, pp. 1365-1375. doi:10.1016/j.fss.2007.08.005

[27] Y.-R. Syau, L.-F. Sugianto and E. S. Lee, "A Class of Semicontinuous Fuzzy Mappings," Applied Mathematics Letters, Vol. 21, No. 8, 2008, pp. 824-827. doi:10.1016/j.aml.2007.09.005

[28] D. P. Filev and R. R. Yager, "A Generalized Defuzzification Method via Bad Distribution,” International Journal of Intelligent Systems, Vol. 6, No. 7, 1991, pp. 687-697. doi:10.1002/int.4550060702

[29] R. Goetschel and W. Voxman, "Elementary Fuzzy Calculus,” Fuzzy Sets and Systems, Vol. 18, No. 1, 1986, pp.
31-43. doi:10.1016/0165-0114(86)90026-6

[30] D. Dubois and H. Prade, "Operations on Fuzzy Numbers,” International Journal of Systems Science, Vol. 9, No. 6, 1978, pp. 613-626. doi:10.1080/00207727808941724

[31] S. Heilpern, "The Expected Value of a Fuzzy Number," Fuzzy Sets and Systems, Vol. 47, No. 1, 1992, pp. 81-86. doi:10.1016/0165-0114(92)90062-9

[32] B. Asady and A. Zendehnam, "Ranking of Fuzzy Numbers by Distance Minimization,” Applied Mathematical Modelling, Vol. 31, No. 11, 2007, pp. 2589-2598.

[33] S. Abbasbandy and B. Asady, "A Note on 'A New Approach for Defuzzification',” Fuzzy Sets and Systems, Vol. 128, No. 1, 2002, pp. 131-132. doi:10.1016/S0165-0114(01)00247-0 